\title{
BMC Genetics reviewer acknowledgement 2014
}

Catherine J Potenski

\section{Contributing reviewers}

The editors of BMC Genetics would like to thank all our reviewers who have contributed to the journal in Volume 15 (2014).

Khaled Abu-Amero

Saudi Arabia

Khaled Abu-Amero

Qatar

Jon Ahlinder

Sweden

Karen Aitken

Australia

Eduard Akhunov

USA

Emidio Albertini

Italy

Andreia Amaral

Portugal

Leif Andersson

Sweden

Toby Andrew

UK

Katja Anttila

Finland

Apostolos Apostolidis

Greece

Agustin Aranda

Spain

Sunil Arora

India
Juan Jose Arranz

Spain

Peter Askjaer

Spain

Giovanna Attene

Italy

Paul Auer

USA

Antonios Augustinos

Greece

David Aylor

USA

Yvonne Badke

Belgium

Emilia Bagnicka

Poland

Guihua Bai

USA

Fernando Sebastian Baldi Rey

Brazil

Gabriel Balmus

UK

Eliane Bandinelli

Brazil

Moinak Banerjee

India

\author{
Claiton Bau \\ Brazil \\ Ryan Baugh \\ USA
}

Esther Becker

UK

Dorothea Bedigian

USA

Piotr Bednarek

Poland

Daniele Bellavia

Italy

Bernhard Benkel

Canada

K.E. Bett

Canada

Mehul Bhakta

USA

Dwaipayan Bharadwaj

India

Mangesh Bhide

Slovakia

Jianmin Bian

China

Bill Black

USA

Correspondence: catherine.potenski@biomedcentral.com

BioMed Central, 233 Spring Street, New York, NY 10013-1578, United States

of America 
Paul Bloor

Colombia

Elizabeth Blue

USA

Lorenzo Bomba

Italy

Gouesnard Brigitte

France

Kevin Brix

Canada

Gudrun Brockmann

Germany

Karl Broman

USA

Richard Brown

UK

Anna Brüniche-Olsen

Australia

Sam Buckberry

Australia

Ana I. Burguete García

Mexico

Mark Burow

USA

Christopher Burtner

USA

Barbara Burwinkel

Germany

Bruno Buzatto

Australia

Diogo Cabral-De-Mello

Brazil

Mario Calus

Netherlands

Samuel Canizales

Mexico

Michele Caraglia

Italy

Ariel Castro

Uruguay

Stanislav Cepica

Czech Republic

Lisa Chakrabarti

UK
Alice H.D. Chan

Singapore

Giriraj Chandak

India

Bian Chao

China

Raquel Chaves

Portugal

Rui Chen

USA

Xiao-Yong Chen

China

Peng Cheng

USA

Li Cheng-Yun

China

Vikram Chhatre

USA

Yoon Shin Cho

Korea, South

Hon Yin Brian Chung

Hong Kong

Ki Wha Chung

Korea, South

Daniel Ciobanu

USA

Leigh Clark

USA

Veryan Codd

UK

Michael H. Crawford USA

Richard Crooijmans

Netherlands

Yuehua Cui

USA

Kehui Cui

China

Dhanjit Kumar Das

India

Sonia Davila

Singapore

Roberta Davoli

Italy
Julie De Backer

Belgium

Thomas Debener

Germany

James Denvir

USA

Giovanni Destro Bisol

Italy

Sangeeta Dhaubhadel

Canada

Antonio Diaz

Venezuela

Roger Doyle

Canada

Tom Druet

Belgium

Qingzhang Du

China

Shiwei Duan

China

Naomi Duijvesteijn

Netherlands

Martina Durner

USA

Nduna Dzimiri

Saudi Arabia

Serge Edme

USA

Mohammed El Mzibri

Morocco

David Ellinghaus

Germany

Ronald Ellis

USA

Ahmed El-Sohemy

Canada

Mauricio Elzo

USA

Malena Erbe

Germany

Nir Eynon

Australia

Patricia Faivre Rampant

France 
Md Fakruddin

Bangladesh

Bin Fan

China

Chao Fang

USA

Justin Faris

USA

Laura Fejerman

USA

Tao Feng

USA

Jesús Fernández

Spain

Luis Fernández

Spain

Rafael Fernández-Muñoz

Spain

Richard Finnell

USA

Lex Flagel

USA

Idhaliz Flores

USA

Laurence Flori

France

Jiri Forejt

Czech Republic

Marina Fortes

Australia

Glen Fox

Australia

Michael Francki

Australia

Elisabetta Frascaroli

Italy

Wenqing Fu

USA

Yong-Bi Fu

Canada

Silvia Fuselli

Italy

Agata Gadaleta

Italy

\author{
Sara Gandini \\ Italy \\ Guimin Gao \\ USA \\ Jonathan Gardner \\ New Zealand \\ Dorian Garrick \\ USA
}

Christiane Gebhardt

Germany

Nicolas Gengler

Belgium

Alessandra Gentile

Italy

Elena Gigli

Italy

Antonio Giordano

USA

Koumudi Godbole

India

Daniel Goedbloed

Germany

Shailendra Goel

India

Luis Gomez-Raya

Spain

Xun Gong

China

Louisa Goumidi

France

Manje Gowda

India

Eric Greer

USA

Christoph Grieder

Switzerland

Martien Groenen

Netherlands

Vince Kornél Grolmusz

Hungary

Sandeep Grover

India

Minxin Guan

China
Rudy Guerra

USA

Beatriz Gutiérrez-Gil

UK

Balazs Györffy

Hungary

Bianca Haase

Australia

Michael Hackenberg

Spain

Dominik Halas

Canada

Christopher Hamm

USA

Janetta Harbron

South Africa

Takeshi Hayashi

Japan

Tao He

USA

Qiuling He

USA

David Heckel

Germany

Laura Hernandez

USA

Alex Hewitt

Australia

Julie Hicks

USA

Paul Hocking

UK

David Holding

USA

Rikard Holmdahl

Sweden

Michael Vaclav Holmes

UK

Shigeru Honda

Japan

Firoz Hossain

India

Liping Hou

USA 
Chris Hoze

France

Zhibin Hu

China

Zhiqiu Hu

Canada

Hailiang Huang

USA

Chun-Hsi Huang

USA

Celine Huber

France

Cornelis J. J. Huijsmans

Netherlands

Kent Hunter

USA

Julie Hussin

Canada

Eveline Ibeagha-Awemu

Canada

Akihiro Ikeda

USA

Ana Insua

Spain

Daniel Irimia

USA

Kristopher Irizarry

USA

Kamel Jabbari

Germany

Luc Janss

Denmark

Chatchawan Jantasuriyarat

Thailand

Bhavanath Jha

India

Baohu Ji

USA

Xueqiu Jian

USA

Bina Joe

USA

Michael Johnson

Australia
Haja Kadarmideen

Denmark

Thomas Källman

Sweden

Guolian Kang

USA

Henry J Kaplan

USA

Orla Keane

Ireland

Ephraim Kenigsberg

Israel

Hasan Khatib

USA

Pawan Khera

USA

Chiea Chuen Khor

Singapore

James Wraith Kijas

Australia

Joomyeong Kim

USA

Jun Kitano

Japan

Vinod Kurungara

India

Arthur Klatt

USA

Robert Klein

USA

Yann Klimentidis

USA

Chris Knight

Denmark

Sara Knott

UK

Michael Hans Kohn

USA

Sulev Koks

Estonia

Naoshi Kondo

Japan

Xiangyin Kong

China
Kon Konstantinov

Australia

Eszter Kotyuk

USA

Sheri Krams

USA

Juozas Kupcinskas

Lithuania

Bassam Lajin

Syria

Suman Lakhanpaul

India

Susan Lamont

USA

Stefano Landi

Italy

Taimour Langaee

USA

Janine Lasalle

USA

Hernan Laurentin

Venezuela

Fabienne Le Provost

France

Matthew Lebo

USA

Andres Legarra

France

Delphine Legrand

France

Sigrid Lehnert

Australia

Gabriel Leitner

Israel

Chun Li

China

Bingshan Li

USA

Chunying Li

USA

Gang Li

USA

Huawei Li

China 
Hui Li

China

Huihui Li

China

Jingmei Li

Singapore

Ming Li

China

Yi Li

Singapore

Ming Li

USA

Rugang Li

USA

Zitong Li

Finland

Xu Lin

China

Huang Ling

China

Matt Littlejohn

New Zealand

Zhanjiang Liu

USA

Po-Ru Loh

USA

Jirong Long

USA

Luciana Lourenco

Brazil

Duc Lu

Canada

Tu Luan

Norway

Pierre Luisi

Spain

Jiangtao Luo

USA

Shiwen Luo

China

Guansheng Ma

China

Xiaochun Ma

China
Peipei Ma

China

Steven Mack

USA

Dylan Maddox

USA

Eva Madrid

Spain

Robert Maier

Australia

Christian Maltecca

USA

Licinio Manco

Portugal

Hideyuki Mannen

Japan

Yoshiro Mano

Japan

Balram Marathi

Philippines

Antonio Marco

UK

Gallegos-Arreola Martha Patricia Mexico

Finian Martin

Ireland

Paula Martins-Lopes

Portugal

Kyonoshin Maruyama

Japan

Sarabjit Mastana

UK

Tara Mcdaneld

USA

Aline Meirhaeghe

France

Alessio Mengoni

Italy

Barbara Migeon

USA

Satoshi Mikawa

Japan

Makiko Mimura

Japan
Ignacy Misztal

USA

Andrew Mitchell

Australia

Toshiaki Mitsui

Japan

Paola Modesto

Italy

Battini Mohan Reddy

India

Grant Morahan

Australia

Takayuki Morisaki

Japan

Michael Mullen

Ireland

Yoshihiro Muneta

Japan

Zuzana Münzbergová

Czech Republic

Joram Mwacharo

UK

Xinzhi Ni

USA

Ezequiel Luis Nicolazzi

Italy

Motohide Nishio

Japan

Gloria Nombela

Spain

Dan Nonneman

USA

Evandro Novaes

Brazil

Gaetano Odierna

Italy

Bermseok Oh

Korea, South

Masafumi Ohtsubo

Japan

Morten Olesen

Denmark

Jose L. Oliver

Spain 
Pablo Orozco-Terwengel

UK

Hakan Ozkan

Turkey

Malliya Gounder Palanichamy

China

Kai-Feng Pan

China

Manish Pandey

India

Josef Patzak

Czech Republic

Benedicte Paus

Norway

Hubert Pausch

Germany

Paulino Pérez

Mexico

Miguel Perez-Enciso

Spain

Andrea Cristina Peripato

Brazil

Hans-Peter Piepho

Germany

Francesc Piferrer

Spain

Elena Pilli

Italy

Pablo Pinedo

USA

Daniel Pinero

Mexico

Tommaso Pippucci

Italy

Eva Pisano

Italy

Odilia Popanda

Germany

Daniel Potaczek

Germany

Giorgio Prantera

Italy

Manoj Prasad

India
Nicolas Puillandre

France

Naveen Puppala

USA

Gao-Feng Qiu

China

Deyun Qiu

Australia

Petr Ráb

Czech Republic

Anthony Raizis

New Zealand

Satyawada Rama

Rao India

Harsh Raman

Australia

Mary Ramnitz

USA

Mojgan Rastegar

Canada

Alan Redd

USA

Giuseppe Reforgiato Recupero Italy

Jun Ren

China

Jonathan Rios

USA

Robert Roberts

Canada

Luiz Rocha

USA

Dana Roldan

Argentina

Megan Rolf

USA

Dirk Roos

Netherlands

Guilherme Rosa

USA

Garry Rosewarne

China

Stephanie Rosse

USA
Yves Rosseel

Belgium

Anna Rita Rossi

Italy

María Susana Rossi

Argentina

Mahdi Saatchi

USA

Andrew Sandford

Canada

Daniele Santi

Italy

Adalberto Santos

Brazil

Shinji Sasazaki

Japan

Shunpei Sato

Japan

Arnold Saxton

USA

Massimo Scandura

Italy

Chris-Carolin Schoen

Germany

Holger Scholz

Germany

Martine Schroyen

USA

Heidi Schwarzenbach

Germany

Günther Schweizer

Germany

Federico Scossa

Italy

Stuart Scott

USA

Bertrand Servin

France

Qiuying Sha

USA

Xueyan Shan

USA

Igor Sharakhov

USA 


\begin{tabular}{|c|c|c|}
\hline $\begin{array}{l}\text { Abhay Sharma } \\
\text { India }\end{array}$ & $\begin{array}{l}\text { Ismo Stranden } \\
\text { Finland }\end{array}$ & $\begin{array}{l}\text { Jeane Visentainer } \\
\text { Brazil }\end{array}$ \\
\hline $\begin{array}{l}\text { Min Shi } \\
\text { USA }\end{array}$ & $\begin{array}{l}\text { Guang Sun } \\
\text { Canada }\end{array}$ & $\begin{array}{l}\text { Marco Volante } \\
\text { Italy }\end{array}$ \\
\hline $\begin{array}{l}\text { Takeshi Shimogiri } \\
\text { Japan }\end{array}$ & $\begin{array}{l}\text { Lidan Sun } \\
\text { China }\end{array}$ & $\begin{array}{l}\text { John Vontas } \\
\text { Greece }\end{array}$ \\
\hline $\begin{array}{l}\text { Toshihiko Shiroishi } \\
\text { Japan }\end{array}$ & $\begin{array}{l}\text { Xiangqing Sun } \\
\text { USA }\end{array}$ & $\begin{array}{l}\text { Shigeharu Wakana } \\
\text { Japan }\end{array}$ \\
\hline $\begin{array}{l}\text { Moore Shoemaker } \\
\text { USA }\end{array}$ & $\begin{array}{l}\text { Akiko Takasuga } \\
\text { Japan }\end{array}$ & $\begin{array}{l}\text { Ronald Walter } \\
\text { USA }\end{array}$ \\
\hline $\begin{array}{l}\text { Alicja Sieminska } \\
\text { Poland }\end{array}$ & $\begin{array}{l}\text { Nicholas Tan } \\
\text { USA }\end{array}$ & $\begin{array}{l}\text { Chunkao Wang } \\
\text { USA }\end{array}$ \\
\hline $\begin{array}{l}\text { Xueling Sim } \\
\text { USA }\end{array}$ & $\begin{array}{l}\text { Zheng-Zheng Tang } \\
\text { USA }\end{array}$ & $\begin{array}{l}\text { Chenguang Wang } \\
\text { USA }\end{array}$ \\
\hline $\begin{array}{l}\text { Domagoj Simic } \\
\text { Croatia }\end{array}$ & $\begin{array}{l}\text { Jeremy Taylor } \\
\text { USA }\end{array}$ & $\begin{array}{l}\text { Haijian Wang } \\
\text { China }\end{array}$ \\
\hline $\begin{array}{l}\text { Kuldeep Singh } \\
\text { India }\end{array}$ & $\begin{array}{l}\text { Chunfa Tong } \\
\text { China }\end{array}$ & $\begin{array}{l}\text { Jin-Jun Wang } \\
\text { China }\end{array}$ \\
\hline $\begin{array}{l}\text { Andrew Singson } \\
\text { USA }\end{array}$ & $\begin{array}{l}\text { Sissades Tongsima } \\
\text { Thailand }\end{array}$ & $\begin{array}{l}\text { Ming Li Wang } \\
\text { USA }\end{array}$ \\
\hline $\begin{array}{l}\text { Robert Smith } \\
\text { Australia }\end{array}$ & $\begin{array}{l}\text { Shaun Townsend } \\
\text { USA }\end{array}$ & $\begin{array}{l}\text { Wen Wang } \\
\text { USA }\end{array}$ \\
\hline $\begin{array}{l}\text { Alex Smith } \\
\text { Canada }\end{array}$ & $\begin{array}{l}\text { Roberto Tuberosa } \\
\text { Italy }\end{array}$ & $\begin{array}{l}\text { Zhong Wang } \\
\text { USA }\end{array}$ \\
\hline $\begin{array}{l}\text { Harold Smith } \\
\text { USA }\end{array}$ & $\begin{array}{l}\text { Meryanne Tumonggor } \\
\text { USA }\end{array}$ & $\begin{array}{l}\text { Xingjun Wang } \\
\text { China }\end{array}$ \\
\hline $\begin{array}{l}\text { Steve Smith } \\
\text { Austria }\end{array}$ & $\begin{array}{l}\text { Takayuki Ueno } \\
\text { Japan }\end{array}$ & $\begin{array}{l}\text { Yaqun Wang } \\
\text { USA }\end{array}$ \\
\hline $\begin{array}{l}\text { Timothy Smith } \\
\text { USA }\end{array}$ & $\begin{array}{l}\text { Guilherme T Valente } \\
\text { Brazil }\end{array}$ & $\begin{array}{l}\text { Zuoheng Wang } \\
\text { USA }\end{array}$ \\
\hline $\begin{array}{l}\text { Petr Smykal } \\
\text { Czech Republic }\end{array}$ & $\begin{array}{l}\text { Johannes Bchm Van Kaam } \\
\text { Italy }\end{array}$ & $\begin{array}{l}\text { Jonas Warringer } \\
\text { Sweden }\end{array}$ \\
\hline $\begin{array}{l}\text { Warren Snelling } \\
\text { USA }\end{array}$ & $\begin{array}{l}\text { Mannis Van Oven } \\
\text { Netherlands }\end{array}$ & $\begin{array}{l}\text { Toshio Watanabe } \\
\text { Japan }\end{array}$ \\
\hline $\begin{array}{l}\text { Hidenobu Soejima } \\
\text { Japan }\end{array}$ & $\begin{array}{l}\text { Annalisa Varriale } \\
\text { Italy }\end{array}$ & $\begin{array}{l}\text { Dirk Wedekind } \\
\text { Germany }\end{array}$ \\
\hline $\begin{array}{l}\text { James Squires } \\
\text { Canada }\end{array}$ & $\begin{array}{l}\text { Monica Vetter } \\
\text { USA }\end{array}$ & $\begin{array}{l}\text { Changshuai Wei } \\
\text { USA }\end{array}$ \\
\hline $\begin{array}{l}\text { Juan Steibel } \\
\text { USA }\end{array}$ & $\begin{array}{l}\text { Johanna Vilkki } \\
\text { Finland }\end{array}$ & $\begin{array}{l}\text { Peng Wei } \\
\text { USA }\end{array}$ \\
\hline $\begin{array}{l}\text { Don Stewart } \\
\text { Canada }\end{array}$ & $\begin{array}{l}\text { Teresa Villarreal-Molina } \\
\text { Mexico }\end{array}$ & $\begin{array}{l}\text { Wen-Hua Wei } \\
\text { UK }\end{array}$ \\
\hline $\begin{array}{l}\text { Patrick Stover } \\
\text { USA }\end{array}$ & $\begin{array}{l}\text { John Vincent } \\
\text { Canada }\end{array}$ & $\begin{array}{l}\text { Olga Wellnitz } \\
\text { Switzerland }\end{array}$ \\
\hline
\end{tabular}


Yangjun Wen

China

Roman Wenne

Poland

Jon White

UK

Klaus Wimmers

Germany

Naomi Wray

Australia

Chih-Chieh Wu

Taiwan

Song Wu

USA

Weiren Wu

China

Xiaowei Wu

USA

Fangming Xie

Philippines

Conghua Xie

China

Chao Xing

USA

Yongzhong Xing

China

Hongyan Xu

USA

Jianlong Xu

China

Shuhua Xu

China
Yaji Xu

USA

Chao-Yie Yang

USA

Jie Yang

USA

Jianzhong Yang

China

Rong-Cai Yang

Canada

Liming Yang

USA

Wenyu Yang

China

Guoyou Ye

Philippines

Guoyou Ye

Australia

Kyung Lim Yoon

Korea, South

Bing Yu

USA

Jianming Yu

USA

Lixing Yuan

China

Gen Hua Yue

Singapore

Junming Yue

USA

Mohamed Zaghloul

Egypt
Robert Zemetra

USA

Weiwei Zhai

Singapore

Haimao Zhan

USA

Ben Zhang

China

Deqiang Zhang

China

Fengyu Zhang

China

Yuan-Ming Zhang

China

Xiquan Zhang

China

Wei Zhao

USA

Yonglan Zheng

USA

Yang Zhiling

China

Igor Zhimulev

Russian Federation

Wei Zhong

China

Chengsong Zhu

USA 